\title{
Pseudotumor Cerebri in a Child With Familial Mediterranean Fever
}

\author{
Faruk İNCECİK, ${ }^{1}$ Özlem M. HERGÜNER, ${ }^{1}$ Mustafa YILMAZ, ${ }^{2}$ Şakir ALTUNBAŞAK ${ }^{1}$ \\ ${ }^{1}$ Department of Pediatric Neurology, Medical Faculty of Çukurova University, Adana, Turkey \\ ${ }^{2}$ Department of Pediatric Immunology, Medical Faculty of Çukurova University, Adana, Turkey
}

\begin{abstract}
Familial Mediterranean fever is an autosomal recessive polysystemic disease characterized by attacks of relapsing and self-limiting fever, peritonitis, pleuritis and arthritis. Neurologic involvement is rare but serious in Familial Mediterranean fever. Headache, seizures, demyelinating lesions, stroke, posterior reversible leukoencephalopathy syndrome, aseptic meningitis, convulsions, and cranial neuropathy have been reported in the literature. In this article, we present the first Familial Mediterranean fever and pseudotumor cerebri association in a child and discuss the pertinent literature. Keywords: Children; familial Mediterranean fever; pseudotumor cerebri.
\end{abstract}

Idiopathic intracranial hypertension (IIH) or pseudotumor cerebri is a well-defined condition with various clinical manifestation. The usual presentations are headache, vomiting, visual disturbances such as papilledema, cranial nerve palsies, or a combination of these signs. ${ }^{1}$

Familial Mediterranean fever (FMF) is an autosomal recessive autoinflammatory disease, presenting with recurrent episodes of fever and polyserositis. Neurological involvement in FMF is rare and shows a wide spectrum, including optic neuritis, recurrent aseptic meningitis, cranial nerve lesions, $\mathrm{IIH}$, ischemic stroke due to coincidental reasons, and subclinical visual evoked potentials abnormalities with demyelinating lesions. ${ }^{2,3}$

Only a few reliable reports are present on the association of FMF and IIH in adults. ${ }^{4,5}$ To our knowledge, there is no report involving children. Thus, in this article, we report a rare case of a child with FMF and IIH. To our knowledge, this is the first report of an association of these two conditions.

\section{CASE REPORT}

A 10-year-old boy presented with a history of frontal headache, vomiting, and diplopia for 10 days. He was known to have FMF (homozygous with M694V mutation) and had been treated with colchicine for one year. Before FMF diagnosis and treatment, his complaints had been recurring two or three times each month for approximately two years. His complaints resolved with colchicine treatment.

On examination, vital signs including blood pressure were normal. His height and weight were also normal for his age. On neurologic examination, he was fully oriented with fluent speech and intact memory. There was bilateral papilledema and a left sixth cranial nerve palsy. The remainder of the physical and neurological examination was unremarkable.

On laboratory examination, routine hematological, biochemical, and urine analyses were normal. A lumbar puncture revealed elevated 
intracranial pressure $\left(450 \mathrm{mmH}_{2} \mathrm{O}\right)$. Patient was not agitated and did not cry during lumbar puncture. Cerebrospinal fluid composition was normal, and cultures were negative. Serologic tests for toxoplasmosis, rubella, cytomegalovirus, herpes simplex virus, Ebstein Barr virus, brucella, salmonella, hepatitis A, B, human immunodeficiency virus, Borrelia burgdorferi, and Mycoplasma pneumoniae were negative. Brain magnetic resonance imaging and magnetic resonance venography studies showed no intracranial lesion or thrombosis. Thus, the patient was diagnosed with $\mathrm{IIH}$. He was started on oral acetazolamide $(20 \mathrm{mg} / \mathrm{kg})$ daily. Further examinations of associated conditions of $\mathrm{IIH}$ including serum levels of vitamin $\mathrm{D}$, vitamin A, thyroid hormones, iron and ferritin levels, antibodies for several viruses, and immunological tests for autoimmune disorders were all normal. After treatment, we observed that his neurological symptoms significantly improved and optic disc edema resolved on ophthalmological examination. Therefore, repeat lumbar puncture was not performed.

\section{DISCUSSION}

Familial Mediterranean fever is an inherited disorder affecting several ethnic groups in the Middle East and Mediterranean countries. The FMF gene, located on chromosome 16p13.3 and symbolized as "MEFV" (for MEditerranean FeVer), encodes a 781 amino acid protein named pyrin. Pyrin is expressed mainly in granulocytes and is thought to be a negative regulator of inflammation. ${ }^{6}$

Although FMF is a multisystem disease, its involvement with central nervous system is not certain. Neurologic involvement in children with FMF is relatively uncommon and rarely described in the pediatric literature. ${ }^{2,7-9}$ Although headaches occur frequently, other manifestations are rare. A large part of these manifestations may be directly related to FMF, its complications, associated diseases, and treatment adverse effects. ${ }^{3}$

Ertekin et al. ${ }^{10}$ described seizures in two of 52 children with FMF. While one of the patients with seizures had electroencephalography abnormality, the other was considered as simple febrile convulsion. In another study, Gedalia and $Z_{a m i r}^{8}$ reported neurologic manifestations in 13 of 101 children with FMF. Of these 13 patients, 10 had headaches during acute episodes of the fever, two had convulsions, and another patient had aseptic meningitis. Kalyoncu et al. ${ }^{2}$ reported 18 patients with neurological signs and detected seven patients with demyelinating lesions, seven patients with cerebrovascular disease, and four patients with reversible posterior leukoencephalopathy syndrome.

In the literature, IIH has been reported in two adult patients with FMF. ${ }^{4,5}$ Gokalp et al. ${ }^{4}$ reported a female patient with concurrent IIH and FMF. Their patient had normal weight and all her relevant laboratory and radiological parameters were also normal. The other patient was described by Kumar. ${ }^{5}$ However, to our knowledge, FMF's association with IIH has not been reported before in children. The pathogenetic mechanism of IIH in FMF is not yet fully understood. However, autoinflammatory mediated injury within the arachnoid villi and the resultant decrease in cerebrospinal fluid absorption and/or thrombotic, vasculitic obliteration of cerebral arteriolar and venous systems are among the most probable mechanisms. ${ }^{3}$

In conclusion, to our knowledge, our patient is the first case of FMF and IIH coexistence in the literature. Although the central nervous system is rarely affected in patients with FMF, neurologic involvement might carry the risk of severe disability. IIH may be a manifestation of FMF in children. Early diagnosis is important for preserving visual functions.

\section{Declaration of conflicting interests}

The authors declared no conflicts of interest with respect to the authorship and/or publication of this article.

\section{Funding}

The authors received no financial support for the research and/or authorship of this article.

\section{REFERENCES}

1. Rangwala LM, Liu GT. Pediatric idiopathic intracranial hypertension. Surv Ophthalmol 2007;52:597-617.

2. Kalyoncu U, Eker A, Oguz KK, Kurne A, Kalan I, Topcuoglu AM, et al. Familial Mediterranean fever 
and central nervous system involvement: a case series. Medicine (Baltimore) 2010;89:75-84.

3. Feld O, Yahalom G, Livneh A. Neurologic and other systemic manifestations in FMF: published and own experience. Best Pract Res Clin Rheumatol 2012;26:119-33.

4. Gökalp HZ, Başkaya MK, Aydin V. Pseudotumor cerebri with familial Mediterranean fever. Clin Neurol Neurosurg 1992;94:261-3.

5. Kumar S. Bilateral disc edema in familial Mediterranean fever. J Clin Diagn Res 2007;1:521-4.

6. French FMF Consortium. A candidate gene for familial Mediterranean fever. Nat Genet 1997;17:25-31.
7. Akman-Demir G, Gul A, Gurol E, Ozdogan H, Bahar $\mathrm{S}$, Oge $\mathrm{AE}$, et al. Inflammatory/demyelinating central nervous system involvement in familial Mediterranean fever (FMF): coincidence or association? J Neurol 2006;253:928-34.

8. Gedalia A, Zamir S. Neurologic manifestations in familial Mediterranean fever. Pediatr Neurol 1993;9:301-2.

9. Yllmaz U, Gülez N, Cubukçu D, Güzel O, Akinci G, Oztürk A. Recurrent peripheral facial palsy in a child with familial Mediterranean fever. Pediatr Neurol 2013;49:289-91.

10. Ertekin V, Selimoğlu MA, Pirim I. Familial Mediterranean fever in a childhood population in eastern Turkey. Pediatr Int 2005;47:640-4. 